\title{
On the angle sum of lines
}

\author{
F. Fodor, V. Vígh and T. Zarnócz
}

\begin{abstract}
What is the maximum of the sum of the pairwise (non-obtuse) angles formed by $n$ lines in the Euclidean 3-space? This question was posed by L. Fejes Tóth in 1959 in [3]. L. Fejes Tóth solved the problem for $n \leq 6$, and proved the asymptotic upper bound $n^{2} \pi / 5$ as $n \rightarrow \infty$. He conjectured that the maximum is asymptotically equal to $n^{2} \pi / 6$ as $n \rightarrow \infty$. The main result of this paper is an upper bound on the sum of the angles of $n$ lines in the Euclidean 3-space that is asymptotically equal to $3 n^{2} \pi / 16$ as $n \rightarrow \infty$.
\end{abstract}

\section{Introduction}

Consider $n$ lines in the $d$-dimensional Euclidean space $\mathbb{R}^{d}$ which all pass through the origin $o$. What is the maximum $S(n, d)$ of the sum of the pairwise (non-obtuse) angles formed by the lines? This question was raised by L. Fejes Tóth in 1959 in 3 for $d=3$. For general $d$, the problem is formulated, for example, in 5 .

The conjectured maximum of the angle sum is attained by the following configuration: Let $n=k \cdot d+m(0 \leq m<d)$, and denote by $x_{1}, \ldots, x_{d}$ the axes of a Cartesian coordinate system in $\mathbb{R}^{d}$. Take $k+1$ copies of each one of the axes $x_{1}, \ldots, x_{m}$, and take $k$ copies of each one of the axes $x_{m+1}, \ldots, x_{d}$. The sum of the pairwise angles in this configuration is

$$
\left[\frac{d(d-1) k^{2}}{2}+m k(d-1)+\frac{m(m-1)}{2}\right] \frac{\pi}{2} .
$$

L. Fejes Tóth stated this conjecture only for $d=3$, however, it is quite natural to extend it to any $d$ (see [5]). To the best of our knowledge, this problem is unsolved for $d \geq 3$.

In the case $d=3$, L. Fejes Tóth [3] proved the conjecture for $n \leq 6$. He determined $S(n, 3)$ for $n \leq 5$ by direct calculation, and he obtained $S(6,3)$ using the recursive upper bound $S(n, 3) \leq n S(n-1,3) /(n-2)$ and the precise value of $S(5,3)$, see pp. 19 in $[3]$. The recursive upper bound and $S(6,3)$ together yield that $S(n, 3) \leq n(n-1) \pi / 5$ for all $n$. We further note 
that L. Fejes Tóth's recursive upper bound on $S(n, 3)$ also holds for $S(n, d)$, that is, $S(n, d) \leq n S(n-1, d) /(n-2)$ for any meaningful $n$ and $d$.

Our main result is summarized in the following theorem.

Theorem 1.1. Let $l_{1}, \ldots, l_{n}$ be lines in $\mathbb{R}^{3}$ which all pass through the origin. If we denote by $\varphi_{i j}$ the angle formed by $l_{i}$ and $l_{j}$, then

$$
\sum_{1 \leq i<j \leq n} \varphi_{i j} \leq\left\{\begin{array}{lll}
\frac{3}{2} k^{2} \cdot \frac{\pi}{2}, & \text { if } & n=2 k, \\
\frac{3}{2} k(k+1) \cdot \frac{\pi}{2}, & \text { if } & n=2 k+1 .
\end{array}\right.
$$

We note that the conjectured maximum for $d=3$ is asymptotically equal to $n^{2} \pi / 6$ as $n \rightarrow \infty$. The upper bound in Theorem 1.1 is asymptotically $3 n^{2} \pi / 16$ as $n \rightarrow \infty$, so it improves on L. Fejes Tóth's bound which is $n^{2} \pi / 5$ as $n \rightarrow \infty$. We also note that if one could prove that $S(8,3)$ is equal to the conjectured value, then combining it with L. Fejes Tóth's recursive upper bound on $S(n, 3)$, one would obtain an upper bound on $S(n, 3)$ that is asymptotically equal to the one in Theorem 1.1 .

We mention that the corresponding problem in which we seek the maximum of the sum of the angles of $n$ rays emanating from the origin of $\mathbb{R}^{d}$ is solved for any $d$ and $n$. This problem was also posed in the same paper of L. Fejes Tóth 3 for $d=3$. The 3-dimensional problem was fully solved as of 1965, see 3, 4, 6 8]. The proof of Nielsen 7] uses a projection averaging argument. We note that this argument can be modified so as to obtain a solution of the general case of the problem for every $n$ and $d$. Our proof of Theorem 1.1 also uses this projection averaging idea, however, the details are much more intricate.

\section{The planar case}

Before we prove Theorem 1.1. we solve the problem in the plane. This result is probably known 5], however, we were unable to find any other reference, thus, we decided to include a short proof for the sake of completeness.

Theorem 2.1. Let $l_{1}, \ldots, l_{n}$ be lines in $\mathbb{R}^{2}$ which all pass through the origin. If we denote by $\varphi_{i j}$ the angle formed by $l_{i}$ and $l_{j}$, then

$$
\sum_{1 \leq i<j \leq n} \varphi_{i j} \leq\left\{\begin{array}{lll}
k^{2} \cdot \frac{\pi}{2}, & \text { if } & n=2 k, \\
k(k+1) \cdot \frac{\pi}{2}, & \text { if } & n=2 k+1 .
\end{array}\right.
$$

Proof. Note that a simple compactness argument guarantees that the maximum of the angle sum exists, and it is attained by some configuration.

Observe that if $l$ and $l^{\prime}$ are two perpendicular lines and $l^{\prime \prime}$ is an arbitrary third line, then the angle sum determined by $l, l^{\prime}$, and $l^{\prime \prime}$ is always $\pi$. This implies that if we have a perpendicular pair in a configuration of lines, then the pair can be freely rotated about the origin while the total sum of the angles remains unchanged.

Let $k=\lfloor n / 2\rfloor$, then $n=2 k$ or $n=2 k+1$. We are going to show that any configuration of $n$ lines can be continuously transformed into a configuration 


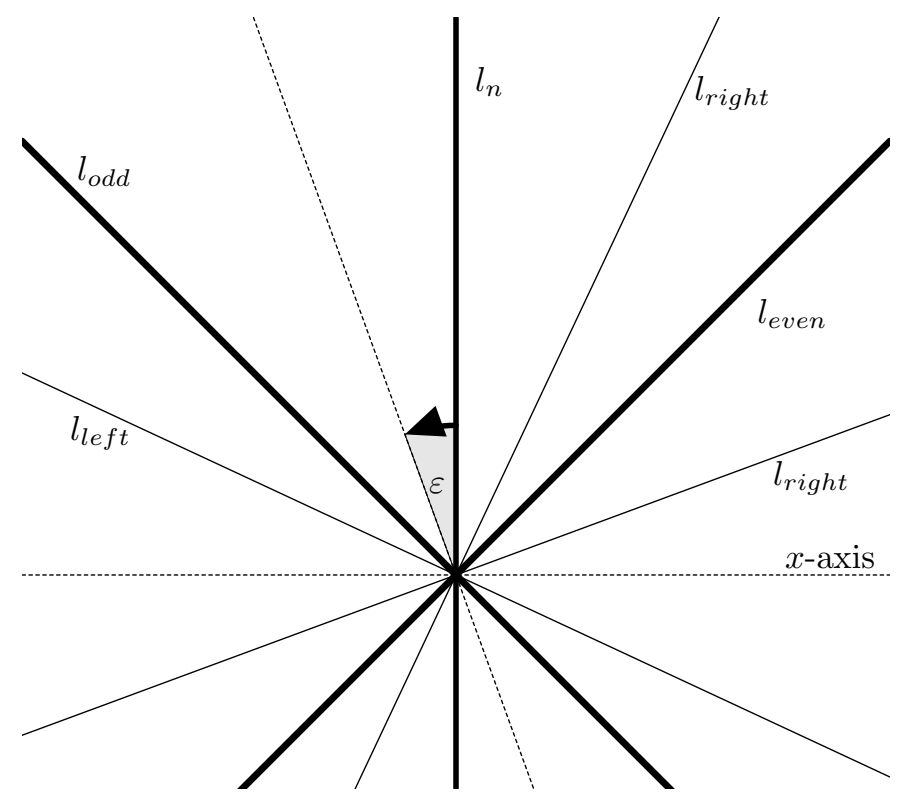

FiguRE 1. Rotating $l_{1}$

that is the disjoint union of $k$ perpendicular pairs (and possibly one remaining line in arbitrary position) such that the angle sum does not decrease during the transformation. This clearly proves Theorem 2.1.

Assume that $\left(l_{1}, l_{2}\right), \ldots,\left(l_{2 m-1}, l_{2 m}\right), m<k$ is a maximal set of pairwise disjoint perpendicular pairs in $l_{1}, \ldots, l_{n}$. During the transformation we will keep each already existing perpendicular pair. By the above observation, we may disregard these pairs as the angle sum of $l_{1}, \ldots, l_{n}$ is independent of their positions.

Let $l_{n}$ be vertical (it coincides with the $y$-axis), see Figure 1. We say that a line $l_{i}$ is to the right of $l_{n}$ if $l_{i}$ is obtained from $l_{n}$ by a rotation about the origin with angle $\alpha$, where $-\pi / 2<\alpha<0$. Similarly, if $0<\alpha<\pi / 2$, then $l_{i}$ is to the left of $l_{n}$. If $l_{i}=l_{n}$, then $l_{i}$ is neither to the left nor to the right of $l_{n}$. By symmetry, we may clearly assume that there are at least as many lines to the right of $l_{n}$ as to the left. The case $l_{2 m+1}=l_{2 m+2}=\ldots=l_{n}$ being obvious, we may assume that there is at least one line to the right of $l_{n}$.

Observe that rotating $l_{n}$ by a small positive angle $\varepsilon>0$, the sum of the angles in $l_{1}, \ldots, l_{n}$ does not decrease. Thus, we may rotate $l_{n}$ until it becomes perpendicular to a line on its right-hand side. In this way, we have created a new perpendicular pair that is disjoint from $\left(l_{1}, l_{2}\right), \ldots,\left(l_{2 m-1}, l_{2 m}\right)$. This completes the proof of Theorem 2.1. 


\section{Proof of Theorem 1.1}

Let $S^{2}$ be the unit sphere of $\mathbb{R}^{3}$ centred at the origin. We denote the Euclidean scalar product by $\langle\cdot, \cdot\rangle$ and the induced norm by $|\cdot|$. For $\mathbf{u}, \mathbf{v} \in S^{2}$, we introduce $\mathbf{v}^{\mathbf{u}}=(\mathbf{u} \times \mathbf{v}) \times \mathbf{u}$, which is the component of $\mathbf{v}$ perpendicular to u. Let $\mathbf{v}_{1}, \mathbf{v}_{2} \in S^{2}$, and let $\varphi=\angle\left(\mathbf{v}_{\mathbf{1}}, \mathbf{v}_{\mathbf{2}}\right)$ denote the angle formed by $\mathbf{v}_{1}, \mathbf{v}_{2}$. Introduce $\varphi^{\mathbf{u}}=\varphi^{\mathbf{u}}\left(\mathbf{v}_{1}, \mathbf{v}_{2}\right)$ for the angle formed by $\mathbf{v}_{1}^{\mathbf{u}}$ and $\mathbf{v}_{2}^{\mathbf{u}}$, and write

$$
\varphi_{*}^{\mathbf{u}}\left(\mathbf{v}_{1}, \mathbf{v}_{2}\right):=\min \left\{\varphi^{\mathbf{u}}\left(\mathbf{v}_{1}, \mathbf{v}_{2}\right), \pi-\varphi^{\mathbf{u}}\left(\mathbf{v}_{1}, \mathbf{v}_{2}\right)\right\}
$$

Let

$$
I\left(\mathbf{v}_{1}, \mathbf{v}_{2}\right)=I(\varphi):=\frac{1}{4 \pi} \int_{S^{2}} \varphi_{*}^{\mathbf{u}}\left(\mathbf{v}_{1}, \mathbf{v}_{2}\right) \mathrm{d} \mathbf{u},
$$

where the integration is with respect to the spherical Lebesgue measure. We will use the following lemma of Fáry 2 .

Lemma 3.1 (Fáry, Lemme 1. on pp. 133 in 2]).

$$
\varphi=\frac{1}{4 \pi} \int_{S^{2}} \varphi^{\mathbf{u}} \mathrm{d} \mathbf{u} \quad \text { for any } 0 \leq \varphi \leq \pi .
$$

We start the proof of Theorem 1.1 with two lemmas.

Lemma 3.2. With the notation introduced above,

$$
I(0)=0 \quad \text { and } \quad I(\pi / 2)=\pi / 3 .
$$

Proof. The statement $I(0)=0$ is clearly true, so we need to calculate $I(\pi / 2)$ only. Let $\mathbf{v}_{1}=(1,0,0), \mathbf{v}_{2}=(0,1,0)$ and define $A=\left\{(x, y, z) \in S^{2} \mid x y \leq 0\right\}$, $A^{C}=\left\{(x, y, z) \in S^{2} \mid x y>0\right\}$, and $A_{+}^{C}=\left\{(x, y, z) \in S^{2} \mid x y>0, x>0\right\}$. Then the following holds

$$
\begin{aligned}
I(\pi / 2) & =\frac{1}{4 \pi} \int_{S^{2}} \varphi_{*}^{\mathbf{u}}\left(\mathbf{v}_{1}, \mathbf{v}_{2}\right) \mathrm{d} \mathbf{u}=\frac{1}{4 \pi} \int_{A} \varphi^{\mathbf{u}} \mathrm{d} \mathbf{u}+\frac{1}{4 \pi} \int_{A^{C}} \pi-\varphi^{\mathbf{u}} \mathrm{d} \mathbf{u} \\
& =\frac{1}{4 \pi} \int_{S^{2}} \varphi^{\mathbf{u}} \mathrm{d} \mathbf{u}-\frac{1}{4 \pi} \int_{A^{C}} \pi-2 \varphi^{\mathbf{u}} \mathrm{d} \mathbf{u} \\
& =\frac{\pi}{2}+\frac{1}{4 \pi} \int_{A^{C}} \pi \mathrm{d} \mathbf{u}-2 \cdot \frac{1}{4 \pi} \int_{A^{C}} \varphi^{\mathbf{u}} \mathrm{d} \mathbf{u} \\
& =\pi-4 \cdot \frac{1}{4 \pi} \int_{A_{+}^{C}} \varphi^{\mathbf{u}} \mathrm{d} \mathbf{u}
\end{aligned}
$$

using Lemma 3.1. Obviously, it is enough to show that

$$
\int_{A_{+}^{C}} \varphi^{\mathbf{u}} \mathrm{d} \mathbf{u}=\frac{2 \pi^{2}}{3} .
$$

Introduce the following spherical coordinates

$$
\mathbf{u}=\mathbf{u}(\theta, \psi)=(\sin \theta \cos \psi, \sin \theta \sin \psi, \cos \theta),
$$


where $0 \leq \theta \leq \pi$ and $0 \leq \psi \leq 2 \pi$. It is easily seen that

$$
\begin{aligned}
\varphi^{\mathbf{u}}\left(\mathbf{v}_{1}, \mathbf{v}_{2}\right) & =\arccos \frac{\left\langle\left(\mathbf{u} \times \mathbf{v}_{1}\right) \times \mathbf{u},\left(\mathbf{u} \times \mathbf{v}_{2}\right) \times \mathbf{u}\right\rangle}{\left|\left(\mathbf{u} \times \mathbf{v}_{1}\right) \times \mathbf{u}\right| \cdot\left|\left(\mathbf{u} \times \mathbf{v}_{2}\right) \times \mathbf{u}\right|} \\
& =\arccos \frac{\left\langle\mathbf{u} \times \mathbf{v}_{1}, \mathbf{u} \times \mathbf{v}_{2}\right\rangle}{\left|\mathbf{u} \times \mathbf{v}_{1}\right| \cdot\left|\mathbf{u} \times \mathbf{v}_{2}\right|} .
\end{aligned}
$$

Straightforward calculations yield that $\mathbf{u} \times \mathbf{v}_{1}=(0, \cos \theta,-\sin \theta \sin \psi)$ and $\mathbf{u} \times \mathbf{v}_{2}=(-\cos \theta, 0, \sin \theta \cos \psi)$, and hence

$$
\begin{aligned}
\left\langle\mathbf{u} \times \mathbf{v}_{1}, \mathbf{u} \times \mathbf{v}_{2}\right\rangle & =-\sin ^{2} \theta \sin \psi \cos \psi, \\
\left|\mathbf{u} \times \mathbf{v}_{1}\right| \cdot\left|\mathbf{u} \times \mathbf{v}_{2}\right| & =\sqrt{\cos ^{2} \theta+\sin ^{4} \theta \sin ^{2} \psi \cos ^{2} \psi} .
\end{aligned}
$$

Thus

$$
\begin{aligned}
\int_{A_{+}^{C}} \varphi^{\mathbf{u}} \mathrm{d} \mathbf{u} & =\int_{0}^{\pi} \int_{0}^{\pi / 2} \arccos \frac{-\sin ^{2} \theta \sin \psi \cos \psi}{\sqrt{\cos ^{2} \theta+\sin ^{4} \theta \sin ^{2} \psi \cos ^{2} \psi}} \cdot \sin \theta \mathrm{d} \psi \mathrm{d} \theta \\
& =2 \cdot \int_{0}^{\pi / 2} \int_{0}^{\pi / 2}\left(\pi-\arctan \frac{\cos \theta}{\sin ^{2} \theta \sin \psi \cos \psi}\right) \cdot \sin \theta \mathrm{d} \psi \mathrm{d} \theta \\
& =\pi^{2}-2 \int_{0}^{\pi / 2} \int_{0}^{\pi / 2} \arctan \frac{\cos \theta}{\sin ^{2} \theta \sin \psi \cos \psi} \cdot \sin \theta \mathrm{d} \theta \mathrm{d} \psi
\end{aligned}
$$

The inner integral in (1) can be directly calculated as follows. Let

$$
\begin{aligned}
g(\theta, \psi)= & \frac{1}{2} \tan \psi \cdot \ln (2 \cos (2 \theta) \cos (2 \psi)+2 \cos (2 \theta)-2 \cos (2 \psi)+6) \\
& +\frac{1}{2} \cot \psi \cdot \ln (-2 \cos (2 \theta) \cos (2 \psi)+2 \cos (2 \theta)+2 \cos (2 \psi)+6) \\
& -\cos \theta \cdot \arctan \frac{\cos \theta}{\sin ^{2} \theta \sin \psi \cos \psi} .
\end{aligned}
$$

One can check by a tedious but straightforward calculation that

$$
\frac{\partial g(\theta, \psi)}{\partial \theta}=\arctan \frac{\cos \theta}{\sin ^{2} \theta \sin \psi \cos \psi} \cdot \sin \theta .
$$

Now, for a fixed $0<\psi<\pi / 2$, we obtain

$$
\begin{aligned}
\int_{0}^{\pi / 2} \arctan & \frac{\cos \theta}{\sin ^{2} \theta \sin \psi \cos \psi} \cdot \sin \theta \mathrm{d} \theta \\
= & \frac{1}{2} \tan \psi \cdot \ln (\cos (\pi-2 \psi)+\cos (\pi+2 \psi)-2 \cos (2 \psi)+4) \\
+ & \frac{1}{2} \cot \psi \cdot \ln (-\cos (\pi-2 \psi)-\cos (\pi+2 \psi)+2 \cos (2 \psi)+4) \\
& -\left[\frac{1}{2} \tan \psi \cdot \ln (\cos (-2 \psi)+\cos (2 \psi)-2 \cos (2 \psi)+8)\right. \\
& \left.+\frac{1}{2} \cot \psi \cdot \ln (-\cos (-2 \psi)-\cos (2 \psi)+2 \cos (2 \psi)+8)-\pi / 2\right]
\end{aligned}
$$




$$
\begin{aligned}
= & \frac{1}{2} \tan \psi \cdot \ln (4(1-\cos (2 \psi)))+\frac{1}{2} \cot \psi \cdot \ln (4(1+\cos (2 \psi))) \\
& \quad+\pi / 2-\frac{\ln 8}{2}(\tan \psi+\cot \psi) \\
= & \frac{1}{2}\left(\pi+\tan \psi \ln \left(\sin ^{2} \psi\right)+\cot \psi \ln \left(\cos ^{2} \psi\right)\right) \\
= & \frac{\pi}{2}+\tan \psi \ln (\sin \psi)+\cot \psi \ln (\cos \psi) .
\end{aligned}
$$

We turn to the outer integral in (1).

$$
\begin{aligned}
\int_{0}^{\pi / 2} & \int_{0}^{\pi / 2} \arctan \frac{\cos \theta}{\sin ^{2} \theta \sin \psi \cos \psi} \cdot \sin \theta \mathrm{d} \theta \mathrm{d} \psi \\
& =\int_{0}^{\pi / 2} \frac{\pi}{2}+\tan \psi \ln (\sin \psi)+\cot \psi \ln (\cos \psi) \mathrm{d} \psi \\
& =\frac{\pi^{2}}{4}+\int_{0}^{\pi / 2} \tan \psi \ln (\sin \psi) \mathrm{d} \psi+\int_{0}^{\pi / 2} \cot \psi \ln (\cos \psi) \mathrm{d} \psi
\end{aligned}
$$

Using the substitution $u=\sin \psi$ in the first integral and $u=\cos \psi$ in the second integral, we obtain that

$$
\int_{0}^{\pi / 2} \tan \psi \ln (\sin \psi) \mathrm{d} \psi=\int_{0}^{\pi / 2} \cot \psi \ln (\cos \psi) \mathrm{d} \psi=\int_{0}^{1} \frac{u \ln u}{1-u^{2}} \mathrm{~d} u .
$$

Integration by parts gives

$$
\int_{0}^{1} \frac{u \ln u}{1-u^{2}} \mathrm{~d} u=\left.\frac{-\ln u \ln \left(1-u^{2}\right)}{2}\right|_{0} ^{1}+\frac{1}{2} \int_{0}^{1} \frac{\ln \left(1-u^{2}\right)}{u} \mathrm{~d} u,
$$

where $\left.\frac{-\ln u \ln \left(1-u^{2}\right)}{2}\right|_{0} ^{1}=0$ by L'Hospital's rule. Now, the substitution $x=u^{2}$ yields

$$
\begin{aligned}
\frac{1}{2} \int_{0}^{1} \frac{\ln \left(1-u^{2}\right)}{u} \mathrm{~d} u & =\frac{1}{4} \int_{0}^{1} \frac{\ln (1-x)}{x} \mathrm{~d} x=\frac{-1}{4} \int_{0}^{1} \frac{\operatorname{Li}_{1}(x)}{x} \mathrm{~d} x \\
& =\frac{-1}{4} \operatorname{Li}_{2}(1)=\frac{-\pi^{2}}{24},
\end{aligned}
$$

where in the last two steps we used the polylogarithm functions $\operatorname{Li}_{s}(z)$ and their well-known properties. For more information on the polylogarithm functions we refer to 9 . This finishes the proof of Lemma 3.2 .

Lemma 3.3. The function $I(\varphi)$ is concave on $[0, \pi / 2]$, and

$$
I(\varphi) \geq 2 \varphi / 3 \quad \text { for } \quad 0 \leq \varphi \leq \pi / 2 .
$$

Before we turn to the proof of Lemma 3.3 , for the sake of completeness, we recall some definitions and a theorem from 1 .

The function $f:[a, b] \rightarrow \mathbb{R}$ is superadditive on $[a, b]$ if for any positive $h<b-a$ and $x \in[a, b-h], f(a+h)-f(a) \leq f(x+h)-f(x)$, cf. Definition 2.2 on pp. 61 in [1]. We call $f$ locally superadditive on $[a, b]$ if for every $x_{0} \in[a, b]$, 


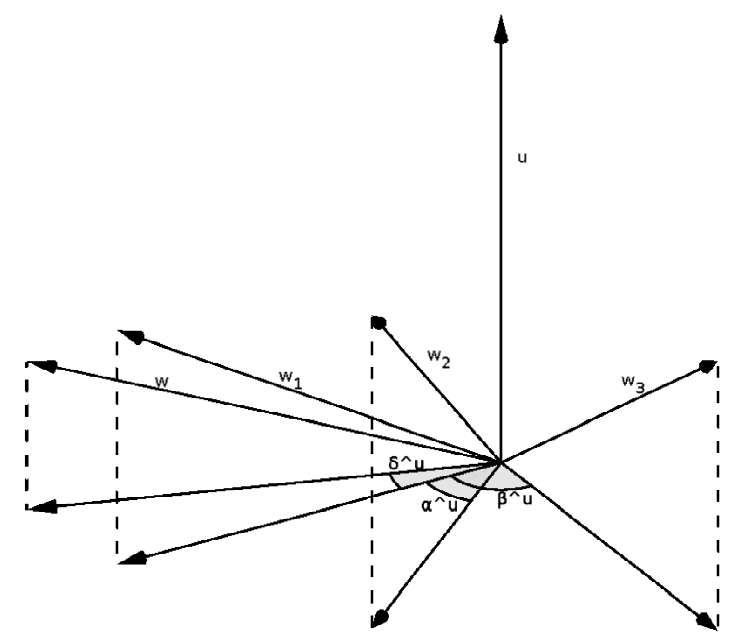

Figure 2. The projection of the angles

there exist arbitrarly small neighborhoods of $x_{0}$ on which $f$ is superadditive, cf. Definition 2.3 on pp. 62 in [1].

Theorem 3.1 (Bruckner, Theorem 3.1. on pp. 62 in [1]). Let $f$ be locally superadditive and differentiable on an interval $[a, b]$, with $f^{\prime}$ continuous almost everywhere in $[a, b]$. Then $f$ is convex.

Proof of Lemma 3.3. Obviously, $I(\varphi)$ is a continuously differentiable function of $\varphi$ on $[0, \pi / 2]$.

Fix $0 \leq \alpha \leq \beta \leq \pi / 2$, a small $0 \leq \delta \leq \pi / 2-\beta$, and a vector $\mathbf{u} \in S^{2}$. Let $\angle(\cdot, \cdot)$ denote the angle formed by two vectors. Choose four coplanar vectors $\mathbf{w}_{1}, \mathbf{w}_{2}, \mathbf{w}_{3}, \mathbf{w} \in S^{2}$ such that $\angle\left(\mathbf{w}_{1}, \mathbf{w}_{2}\right)=\alpha, \angle\left(\mathbf{w}_{1}, \mathbf{w}_{3}\right)=\beta, \angle\left(\mathbf{w}_{1}, \mathbf{w}\right)=\delta$, $\angle\left(\mathbf{w}, \mathbf{w}_{2}\right)=\alpha+\delta$, and $\angle\left(\mathbf{w}, \mathbf{w}_{3}\right)=\beta+\delta$, see Figure 2, As before, we use the abbreviations $\alpha^{\mathbf{u}}=\alpha^{\mathbf{u}}\left(\mathbf{w}_{1}, \mathbf{w}_{2}\right)$ and $\alpha_{*}^{\mathbf{u}}=\alpha_{*}^{\mathbf{u}}\left(\mathbf{w}_{1}, \mathbf{w}_{2}\right)$, and similarly for the other angles.

We claim that

$$
(\alpha+\delta)_{*}^{\mathbf{u}}-\alpha_{*}^{\mathbf{u}} \geq(\beta+\delta)_{*}^{\mathbf{u}}-\beta_{*}^{\mathbf{u}} .
$$

To prove (3), we write the left-hand side, and, respectively, the righthand side as follows:

$$
(\alpha+\delta)_{*}^{\mathbf{u}}-\alpha_{*}^{\mathbf{u}}= \begin{cases}-\delta^{\mathbf{u}}, & \text { if } \quad \alpha^{\mathbf{u}}>\pi / 2 \\ \pi-2 \alpha^{\mathbf{u}}-\delta^{\mathbf{u}}, & \text { if } \quad \alpha^{\mathbf{u}} \leq \pi / 2 \text { and }(\alpha+\delta)^{\mathbf{u}}>\pi / 2 \\ \delta^{\mathbf{u}}, & \text { if } \quad(\alpha+\delta)^{\mathbf{u}} \leq \pi / 2\end{cases}
$$


and

$$
(\beta+\delta)_{*}^{\mathbf{u}}-\beta_{*}^{\mathbf{u}}= \begin{cases}-\delta^{\mathbf{u}}, & \text { if } \beta^{\mathbf{u}}>\pi / 2 \\ \pi-2 \beta^{\mathbf{u}}-\delta^{\mathbf{u}}, & \text { if } \beta^{\mathbf{u}} \leq \pi / 2 \text { and }(\beta+\delta)^{\mathbf{u}}>\pi / 2 \\ \delta^{\mathbf{u}}, & \text { if }(\beta+\delta)^{\mathbf{u}} \leq \pi / 2\end{cases}
$$

To show (3), we consider three cases as in (4). If $\alpha^{\mathbf{u}}>\pi / 2$, then $\beta^{\mathbf{u}}>$ $\pi / 2$, and equality holds in (3). If $\alpha^{\mathbf{u}} \leq \pi / 2$ and $(\alpha+\delta)^{\mathbf{u}}>\pi / 2$, then $(\beta+\delta)^{\mathbf{u}}>$ $\pi / 2$, and either the first or the second case applies in $(5)$. Now, $\pi-2 \alpha^{\mathbf{u}}-\delta^{\mathbf{u}} \geq$ $-\delta^{\mathbf{u}}$ is equvivalent to $\alpha^{\mathbf{u}} \leq \pi / 2$, thus it holds true. Also, from $\alpha^{\mathbf{u}} \leq \beta^{\mathbf{u}}$, it follows that $\pi-2 \alpha^{\mathbf{u}}-\delta^{\mathbf{u}} \geq \pi-2 \beta^{\mathbf{u}}-\delta^{\mathbf{u}}$, as claimed. The only case that remains to be checked is when $(\alpha+\delta)^{\mathbf{u}} \leq \pi / 2$, and thus $(\alpha+\delta)_{*}^{\mathbf{u}}-\alpha_{*}^{\mathbf{u}}=\delta^{\mathbf{u}}$. If, in (5), the first or the third case applies, then the inequality in (3) clearly holds. Thus, we only need to consider the case when $(\beta+\delta)^{\mathbf{u}}>\pi / 2$. Then $\delta^{\mathbf{u}}>\pi-2 \beta^{\mathbf{u}}-\delta^{\mathbf{u}}$, which finishes the proof of $(3)$.

Since (3) holds true for any unit vector $\mathbf{u} \in S^{2}$, it follows that for any $0 \leq \alpha \leq \beta \leq \pi / 2$, and $0 \leq \delta \leq \pi / 2-\beta$, we have

$$
I(\alpha+\delta)-I(\alpha) \geq I(\beta+\delta)-I(\beta) .
$$

Hence $-I$ is superadditive on any subinterval of $[0, \pi / 2]$, and thus it satisfies all the conditions of Theorem 3.1 on the interval $[0, \pi / 2]$. It follows that $-I$ is convex, and so $I$ is concave, as stated. Finally, the inequality (2) is a simple consequence of Lemma 3.2 and of the concavity of $I$. This completes the proof of Lemma 3.3 .

Proof of Theorem 1.1. Consider the lines $l_{1}, \ldots, l_{n}$, and a vector $\mathbf{u} \in S^{2}$. Let $S$ be the plane through the origin with normal vector $\mathbf{u}$, and let $l_{i}^{\prime}$ denote the orthogonal projection of the line $l_{i}$ onto $S$. We denote by $\varphi_{i j}^{\mathbf{u}}$ the (non-obtuse) angle formed by $l_{i}^{\prime}$ and $l_{j}^{\prime}$. Applying (2), we obtain that

$$
\begin{aligned}
\frac{1}{4 \pi} \int_{S^{2}} \sum_{1 \leq i<j \leq n} \varphi_{i j}^{\mathbf{u}} \mathrm{d} u & =\sum_{1 \leq i<j \leq n} \frac{1}{4 \pi} \int_{S^{2}} \varphi_{i j}^{\mathbf{u}} \mathrm{d} u \\
& \geq \sum_{1 \leq i<j \leq n} 2 \varphi_{i j} / 3=\frac{2}{3} \sum_{1 \leq i<j \leq n} \varphi_{i j} .
\end{aligned}
$$

Therefore, there exists a $\mathbf{u}_{0} \in S^{2}$ with the property

$$
\sum_{1 \leq i<j \leq n} \varphi_{i j}^{\mathbf{u}_{0}} \geq \frac{2}{3} \sum_{1 \leq i<j \leq n} \varphi_{i j}
$$

Finally, Theorem 2.1 implies that

$$
\sum_{1 \leq i<j \leq n} \varphi_{i j}^{\mathbf{u}_{0}} \leq\left\{\begin{array}{lll}
k^{2} \cdot \frac{\pi}{2}, & \text { if } & n=2 k \\
k(k+1) \cdot \frac{\pi}{2}, & \text { if } & n=2 k+1
\end{array}\right.
$$

which completes the proof of Theorem 1.1 


\section{Acknowledgements}

This paper was supported in part by TÁMOP-4.2.2.B-15/1/KONV-20150006. F. Fodor wishes to thank the MTA Alfréd Rényi Institute of Mathematics of the Hungarian Academy of Sciences, where this work was done while he was a visiting researcher. V. Vígh was supported by the János Bolyai Research Scholarship of the Hungarian Academy of Sciences.

\section{References}

[1] A. M. Bruckner, Some relationships between locally superadditive functions and convex functions, Proc. Amer. Math. Soc. 15 (1964), 61-65.

[2] I. Fáry, Sur la courbure totale d'une courbe gauche faisant un nœud, Bull. Soc. Math. France 77 (1949), 128-138 (French).

[3] L. Fejes Tóth, Über eine Punktverteilung auf der Kugel, Acta Math. Acad. Sci. Hungar 10 (1959), 13-19 (German).

[4] O. Frostman, A theorem of Fáry with elementary applicatons, Nordisk Mat. Tidskr. 1 (1953), 25-32, 64.

[5] ht tp: //ma thoverfl ow.net /questions/173712/maxi mum-s um-of-ang les-bet we en $-n-l$ in es Last accessed: July 30, 2015.

[6] H. Larcher, Solution of a gemetric problem by Fejes Tòth, Michigan Math. J. 9 (1962), $45-51$.

[7] F. Nielsen, On the sum of the distances between $n$ points on a sphere., Nordisk Mat. Tidskr. 13 (1965), 45-50 (Danish).

[8] G. Sperling, Lösung einer elementargeometrischen Frage von Fejes Tóth, Arch. Math. 11 (1960), 69-71 (German).

[9] D. Zagier, The dilogarithm function in geometry and number theory, Number theory and related topics (Bombay, 1988), Tata Inst. Fund. Res. Stud. Math., vol. 12, Tata Inst. Fund. Res., Bombay, 1989, pp. 231-249.

F. Fodor

Department of Geometry, Bolyai Institute, University of Szeged, Aradi vértanúk tere 1, 6720 Szeged, Hungary, and Department of Mathematics and Statistics, University of Calgary, Canada

e-mail: fodorf@math.u-szeged.hu

V. Vígh

Department of Geometry, Bolyai Institute, University of Szeged, Aradi vértanúk tere 1, 6720 Szeged, Hungary

e-mail: vigvik@math.u-szeged.hu

T. Zarnócz

Department of Geometry, Bolyai Institute, University of Szeged, Aradi vértanúk tere 1, 6720 Szeged, Hungary

e-mail: tzarnocz@math.u-szeged.hu 